\title{
CUTANEOUS LEISHMANIASIS: A CASE REPORT OF A DIAGNOSTIC DILEMMA
}

\author{
Prajwal Pudasaini ${ }^{1}$ \\ ${ }^{1}$ Gandaki Medical College Teaching Hospital and Research Center Pvt Ltd
}

September 24, 2021

\begin{abstract}
Cutaneous Leishmaniasis (CL) is the most prevalent clinical form of leishmaniasis. CL is difficult for the clinicians to diagnose because of the rarity of the disease and non-specific presentation. As CL is rare and given the limitation of available diagnostic modalities in a resource poor setting, diagnosis can be confusing.
\end{abstract}

\section{CUTANEOUS LEISHMANIASIS: A CASE REPORT OF A DIAGNOstic DILEMMA}

Key Words : Cutaneous Leishmaniasis, Fine Needle Aspiration Cytology

Word count: 455 figure:1

Pudasaini $\mathrm{P}^{1}$, Pudasaini $\mathrm{P}^{2}$

${ }^{1}$ Department of Dermatology, Gandaki Medical College Teaching Hospital (GMCTH), Pokhara, Nepal, ${ }^{2}$ Department of General Surgery, Kathmandu Medical College Teaching Hospital (KMCTH)

\section{Authors:}

Dr. Prajwal Pudasaini, Dr. Prashanta Pudasaini

Department of Dermatology Gandaki Medical College Teaching Hospital (GMCTH), Pokhara, Nepal. Email: prajwalpudasaini@gmail.com Phone no: +9779849447266

Conflict of interest : None

Key clinical message: Cutaneous Leishmaniasis (CL) is the most prevalent clinical form of leishmaniasis and is caused by vector borne protozoan parasite. Variation in diagnostic accuracy exists. A 54-year-old female farmer by occupation presented with lesion over right thigh for 8 months.

Lesion evolved over period of 2-3 months and progressed to form ulcer with surrounding redness. On examination, solitary plaque with crateriform ulcer $3^{*} 2 \mathrm{~cm}$ in size roughly oval in shape was present. Ulcer floor was moist, smooth shiny with serous discharge and well defined raised erythematous margin was present. Biopsy was done which showed features suggestive of lupus vulgaris, for which Anti Tubercular Treatment (ATT) was started. There was persistence of ulcer despite 4 months of ATT, for which diagnosis was reconsidered and Fine Needle Aspiration Cytology (FNAC) was performed. FNAC showed numerous intra and extracellular amastigotes suggestive of Leishmaniasis which was treated with complete disappearance of ulcer over 4 months.

\section{Abstract}

Background : Cutaneous Leishmaniasis (CL) is the most prevalent clinical form of leishmaniasis and is caused by vector borne protozoan parasite. ${ }^{1}$ Variation in diagnostic accuracy exists between different 
parasitological and histopathological examination. ${ }^{2}$ Test of choice for diagnosis depends not only on the sensitivity and specificity of a test, but also the availability, especially in a resource poor setting. CL is difficult for the clinicians to diagnose because of the rarity of the disease and non-specific presentation. Here we report a rare case report of CL.

Observation : A 54-year-old female from Gorkha, farmer by occupation with frequent outdoor activities, presented with lesion over right thigh for 8 months. Initially single pinhead sized, soft, raised lesion with brownish red color was noted over right thigh that increased in size with crust formation. Lesion evolved over period of 2-3 months and progressed to form ulcer with surrounding redness. On examination, solitary plaque with crateriform ulcer $3^{*} 2 \mathrm{~cm}$ in size roughly oval in shape was present over the right thigh, upper third aspect in the lateral part, approx.15cm from Anterior Superior Iliac Spine (ASIS). Ulcer floor was moist, smooth shiny with serous discharge and well defined raised erythematous margin was present. Biopsy was done which showed features suggestive of lupus vulgaris, for which Anti Tubercular Treatment (ATT) was started. There was persistence of ulcer despite 4 months of ATT, for which diagnosis was reconsidered and Fine Needle Aspiration Cytology (FNAC) was performed. FNAC showed numerous intra and extracellular amastigotes suggestive of Leishmaniasis which was treated with complete disappearance of ulcer over 4 months.

Discussion : As CL is rare and given the limitation of available diagnostic modalities in a resource poor setting, diagnosis can be confusing. Diagnosis can be made with good clinical acumen and FNAC in an endemic area. ${ }^{3}$ With proper diagnosis overall prevalence of the disease can be estimated and clinical therapeutic trials can be performed with timely prevention of mucocutaneous complications.

\section{Bibliography:}

1. Cutaneous leishmaniasis: current and future management. Hepburn NC Expert Rev Anti Infect Ther. 2003 Dec; 1(4):563-70.

2. Current diagnosis and treatment of cutaneous and mucocutaneous leishmaniasis. Goto H, Lindoso JA Expert Rev Anti Infect Ther. 2010 Apr; 8(4):419-33.

3. Use of fine needle aspiration cytology in the diagnosis of cutaneous leishmaniasis: a comparison with the conventional scraping method.Hosseinzadeh M, Omidifar N, Lohrasb MH Trop Doct. 2012 Apr; 42(2):112-3.

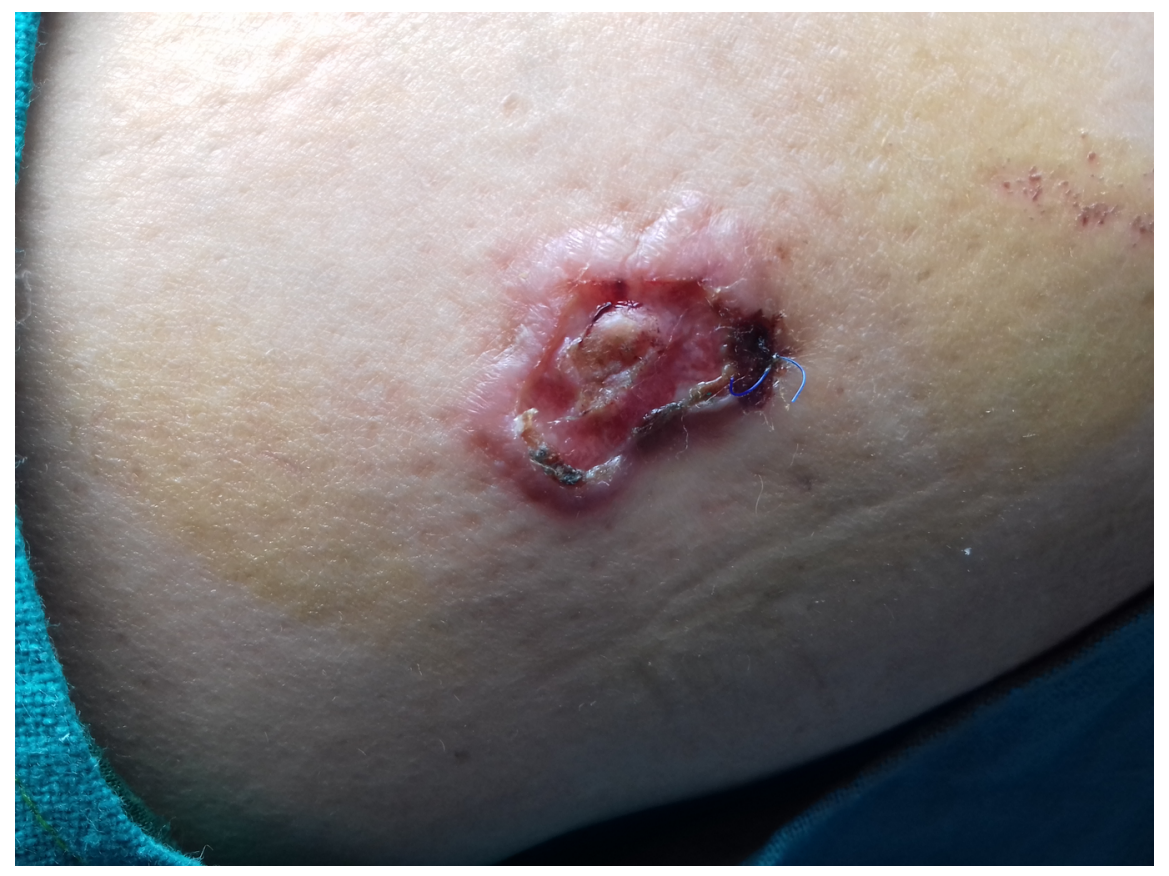

